\title{
Complementary Perfect Domination Number of Regular Graphs
}

\section{Vipin Kumar}

KIET, Ghaziabad, India
Ankit Verma

KIET, Ghaziabad, India

\author{
Shashank Bharadwaj \\ KIET, Ghaziabad, India
}

\begin{abstract}
In this paper authors describe domination number of regular graphs as well as complimentary perfect domination number and induced complimentary perfect domination number, denoted by cpd and iced. Let $\mathrm{G}(\mathrm{v}, \mathrm{e})$ be a graph with ' $\mathrm{n}$ ' vertices and 'e' edges then these are denoted by $X_{c p}(G)$ .and $X_{i c p}(G)$.In this paper we describe, How to calculate $X_{c p}(G)$.and $X_{i c p}(G)$ of regular graphs. Authors characterize 2 regular graphs with $X_{i c p}$ and 3 regular graphs with $X_{i c p}$ and describe an upper limit for number of vertices in d-regular graph. In the end of the paper we characterize all the d-regular graphs with $X_{i c p}(G)$ and practical utilization of cpd and ipcd.
\end{abstract}

\section{Keywords}

Domination number, representation of two graphs with one, cpd and ipcd of regular graph, d-regular.

\section{INTRODUCTION}

In this modern age of science and pacific root of computer life, so many scholars are doing hard work in the domination theory. In this paper we plan a structure of traffic control system with regular graphs and explain how it is used. Because of it more than lacks Rs. are spent on the flyover of a crossing.

Let $\mathrm{G}(\mathrm{v}, \mathrm{e})$ be a regular graph with $\mathrm{n}$ vertices and e edges that means Graph is finite, simple undirected. The number of vertices is denoted by $V_{1}, V_{2}, V_{3}, \ldots, V$, . A lot of work has been done on domination sets and domination number. Different type of domination sets and domination number has been introduced and studied by several authors. [1][4][5][7][10] Paulraj Joseph and Arumugam S have found the relationship between domination number and connectivity in graph as well as domination number and coloring in graphs.

In the same way Paulraj J, Joseph and Mehadevan [11] have introduced the concept of complimentary perfect domination number but they fail to calculate cpd and icpd of regular graphs.

Avadayappan and Senthil Kumar have proved that for any graph $\mathrm{G}$ and a vertex $\mathrm{V}$ of $\mathrm{G}$. $X_{c p}\left(G_{m}(v)\right)=X_{c p}(G)+m$, where $G_{m}(v)_{\text {is the }}$ graph obtained from $\mathrm{G}$ by identification of the center vertex of a star graph $K_{i, m}$ at a vertex V.

\subsection{Domination Sets}

A subset $\mathrm{S}$ of vertices is known domination set of $\mathrm{G}$ if every vertex in $\mathrm{V}-\mathrm{S}$ is adjacent to at least one vertex in $\mathrm{S}$.

\subsection{Domination Number}

Domination number of a graph $\mathrm{G}$ is the minimum cardinality taken over all dominating set in $\mathrm{G}$ and denoted by $\mathrm{X}(\mathrm{G})$.

\subsection{Complementary Perfect Domination Number}

The complimentary perfect domination number of graph $\mathrm{G}$ is the minimum cardinality taken over all complementary perfect domination sets in $\mathrm{g}$ and denoted by $X_{c p}(G)$

\subsection{Induced Perfect Domination Number}

The minimum cardinality taken over all the induced perfect Dominaiton sets of $\mathrm{G}$ is known as induced complementary perfect domination number of $\mathrm{G}$ and denoted by $X_{i c p}(G)$ An induced complementary perfect domination set of a graph $\mathrm{G}$ (icpd-set) is a domination set of $\mathrm{G}$ such that induced sub $\operatorname{graph}(\mathrm{v}-\mathrm{s})$ has only independent edges. Any ipcd-set with $X_{i c p}$ elements is known as $X_{i c p}$-set of G.

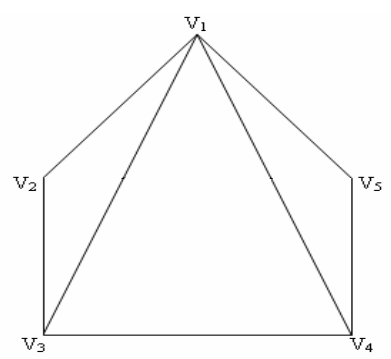

Fig1.1

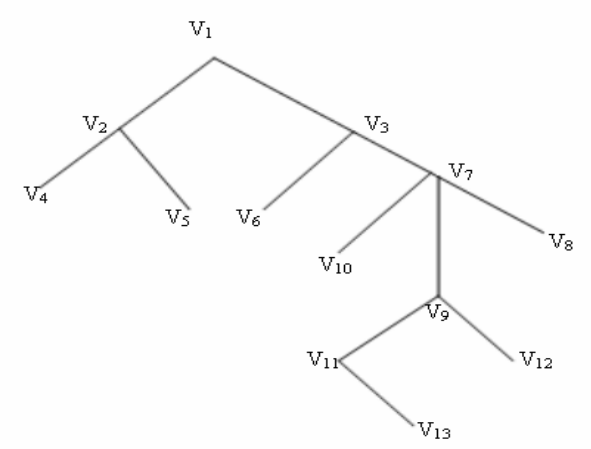

Fig 1.2 
In fig $1.1 X_{c p}(G)=1$ and $X_{i c p}=3$

Here $\mathrm{S}=\left\{V_{1}\right\}$ is the $X_{c p}$-set and $\mathrm{S}=\left\{V_{1}, V_{2}, V_{3}\right\}$ is a $X_{i c p}$-set as well as fig 1.2 has $X_{c p}=8$ and $X_{i c p}=9$ Here $\mathrm{S}=\left\{V_{2}, V_{5}, V_{6}, V_{8}, V_{10}, V_{12}, V_{13}\right\}$ is $X_{c p}$ - set and $\mathrm{S}=\left\{V_{3}, V_{5}, V_{6}, V_{8}, V_{10}, V_{11}, V_{12}, V_{13}\right\}$ is a $X_{i c p}$-set.

\section{NOTATIONS}

$\mathrm{G}(\mathrm{v}, \mathrm{e}) \rightarrow$ A graph with $\mathrm{n}$ vertices and e edges.

$\mathrm{n}$-regular graph $\rightarrow$ A graph $\mathrm{G}$ is said to be $\mathrm{n}-$ regular if degree of Each vertex is n.

$\mathrm{d}(\mathrm{v}) \rightarrow$ Degree of vertex $\mathrm{V}$ in $\mathrm{G}$.

$\Delta \rightarrow$ Maximum degree in $\mathrm{G}$.

$X_{c p}(G) \rightarrow$ Complimentary perfect domination number of

G.

$X_{i c p}(G) \rightarrow$ Induced complimentary perfect domination number of $\mathrm{G}$.

[x] $\rightarrow$ Greatest number, which is at least $\mathrm{x}$.

$K_{n} \rightarrow$ Complete graph with n vertices.

$C_{n} \rightarrow$ Cyclic on $n$ vertices.

$\langle\mathrm{S}\rangle$ or $(\mathrm{S}) \rightarrow$ Induced sub graph of $\mathrm{G}$

$\mathrm{R} \rightarrow$ Chromatic number of a graph.

$K_{3,3}$

$\rightarrow$ Bipartite graph.

\subsection{Assumptions / Observation}

1. For any Graph, $n \geq 2 X_{\text {icp }}(G)=n$ iff $\mathrm{G}$ is a star.

2. $X_{i c p}(G)=1 \Leftrightarrow \mathrm{G}$ is isomorphic to $K_{1} \vee m K_{2}, m \geq 1$

3. Any Icpd - set of $\mathrm{G}$ must contain all the Pendant vertex of $\mathrm{G}$.

4. If G is not star then $\left[\frac{n}{\Delta+1}\right] \leq X_{i c p} \leq n-2$

$$
\text { 5. } X_{i c p}\left(K_{n}\right)=n-2 \forall n \geq 3
$$

\section{COMPLEMENTARY PERFECT DOMINATION NUMBER OF REGULAR GRAPHS}

This section authors try to fill some gap by solving some theorem and its characterization, all cycles of 2-regular graph with $X_{c p}=\alpha$ and 3-regular graphs with $X_{i c p}=\alpha=2$

\section{Theorem 1.1}

$\mathrm{G}$ is 2-regular graph with

$$
X_{c p}(G)=r \Leftrightarrow G \cong c_{n}, n=4,5,6,7,9
$$

Proof: - Given that G is 2-regular i.e. 2-regular is a cycle. $\Rightarrow \quad$ Cycle may be even or odd

Even cycle have $r=2$ and odd cycle have $r=3$.

$$
\begin{array}{ll}
\Rightarrow & X_{c p}=r \\
\Rightarrow & X_{c p}=2 \text { or } 3
\end{array}
$$

Now let $X_{c p}=r=2$ then

$$
2=X_{c p} \geq\left[\frac{n}{\Delta+1}\right]=\left[\frac{n}{3}\right]
$$

Therefore $n \leq 6$

Hence $G \cong C_{4}$ or $C_{6}$

In the same way

$$
\begin{gathered}
X_{c p}=r=3 \\
3=V_{c p} \geq\left[\frac{n}{\Delta+1}\right]=\left[\frac{n}{3}\right] \\
\Rightarrow P \leq 9_{\text {And }} G \cong C_{5 \text { or }} C_{7 \text { or }} C_{9}
\end{gathered}
$$

Conversely,

$$
G \cong C_{n}, n=4,5,6,7,9
$$

$\Rightarrow$ There exist some circuits of even and odd length.

$\Rightarrow \mathrm{G}$ is 2-regular with $X_{c p}=r$

\section{Theorem 1.2}

G is a 3 regular graph with $X_{c p}=r=2$ if and only if $G \cong K_{3,3}$

Proof: - First assuming that $\mathrm{G}$ is 3-regular graph with

$$
X_{c p}=r=2 \text { and prove that } G \cong K_{3,3}
$$

G be 3-regular graph with $X_{c p}=r=2$ than

$$
\begin{aligned}
2=X_{c p} & \geq\left[\frac{n}{\Delta+1}\right]=\left[\frac{n}{3}\right] \\
& \Rightarrow n \leq 8 \\
& \Rightarrow \text { but } \mathrm{G} \text { is 3-regular } \\
& \Rightarrow \mathrm{n}=4,5,6 \text { or } 8 \\
& \Rightarrow n \neq 4
\end{aligned}
$$

Because $\vec{K}_{4}$ is the only 3 -regular graph on 4 -vertices of which $r=4$.

Case I: $-\mathrm{n}=6$

$\Rightarrow \mathrm{n}=6$ or 8 i.e. there are 2 case.

Let $V_{1}, V_{2}, V_{3}, V_{4}, V_{5}, V_{6}$ be the six vertices of G.

Let $\mathrm{S}=\left\{V_{1}, V_{2}\right\}$ be $X_{c p}$ - set then $\langle\mathrm{V}$-S $\rangle$ should have two independent edges say $V_{3} V_{4}$ and $V_{5} V_{6}$. Since $\mathrm{r}=2$, G has no triangle and hence both $V_{3}$ and $V_{4}$ or $V_{5}$ and $V_{6}$ 
cannot be adjacent to $V_{1}$ i.e. $V_{1}$ is adjacent to at most 2 nonadjacent vertices of V.S. but $\mathrm{G}$ is 3-regular with $\mathrm{r}=2$ therefore $V_{1}$ is adjacent to $V_{2}$ and to exactly non-adjacent vertices of V-S say $V_{4}$ and $V_{6}$

Similarly $V_{2}$ is Adjacent to $V_{1}$ and to exactly two nonadjacent vertices of $\mathrm{V}-\mathrm{S}$ say $V_{3}, V_{5}$. Also a vertex in $\langle\mathrm{V}-\mathrm{S}\rangle$ cannot be adjacent to both $V_{1}$ and $V_{2}$. Keeping all above calculation the resulting graph is nothing but $K_{3,3}$ as shown in fig.

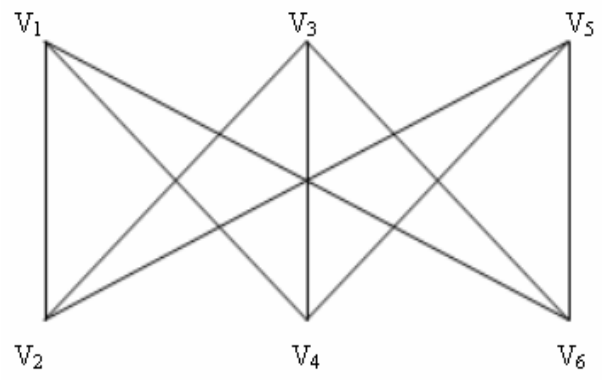

OR

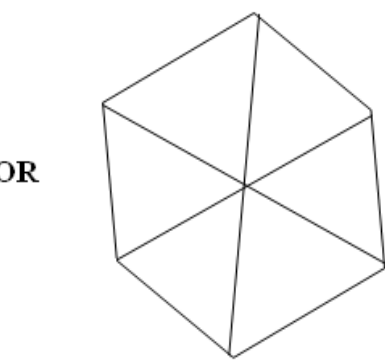

Case II: - If $\mathrm{n}=8$

Let $V_{n} \in G \forall n=1,2, \ldots \ldots, 8$ with $X_{c p}=2$

Let $\mathrm{S}=\left\{V_{1}, V_{2}\right\}$ be a $X_{c p}-$ set . As G is A 3-reglarar graph and $\mathrm{S}$ is dominating set in $\mathrm{V}-\mathrm{S}, V_{1}$ and $V_{2}$ are adjacent to exactly three different vertices in $\mathrm{V}$-S.

Let

$$
R\left(V_{1}\right)=\left\{V_{3}, V_{5}, V_{7}\right\} \text { and }
$$

$R\left(V_{2}\right)=\left\{V_{4}, V_{6}, V_{7}\right\}$. Since $\mathrm{r}=2, \mathrm{G}$ has no triangle and $V_{3}, V_{5}, V_{7}$ are independent. Similarly $V_{4}, V_{6}, V_{8}$ are independent.

Now $\langle\mathrm{V}-\mathrm{S}\rangle$ is 2-regular bipartite graph with $(\mathrm{U}, \mathrm{V})$ Where $\mathrm{U}=\left\{V_{3}, V_{5}, V_{7}\right\}$ and $\mathrm{V}=\left\{V_{4}, V_{6}, V_{7}, V_{8}\right\} \Rightarrow\langle\mathrm{V}-\mathrm{S}\rangle$ is 2regular with six vertices.

Here resulting graph shown as
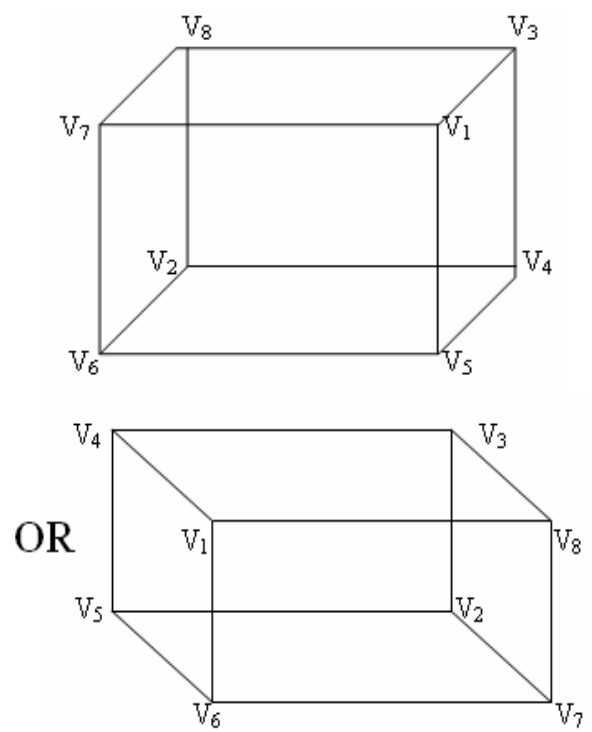

conversely, by theorem we know that r=3 of $K_{3,3}$ r=m for $K_{3,3}, \mathrm{~m}>$ b

\section{INDUCED PERFECT DOMINATION} NUMBER OF REGULAR GRAPH

In this section, Author characterizes all the d-regular graphs with minimum possible induced complimentary perfect domination number.

\section{Theorem 1.3}

If $\mathrm{G}$ is a d-regular graph then $X_{i c p}(G) \geq d-1$ and $n \leq X_{i c p}\left(\frac{2 d-1}{d-1}\right)$

Proof: - Let S be a set $X_{i c p}-s e t$ in G. then all the vertices in $\langle\mathrm{V}-\mathrm{S}\rangle$ have degree 1 .

$$
\text { (From definition of } X_{i c p} \text { ) }
$$

Since the Graph is d-regular every vertex in $\langle\mathrm{V}-\mathrm{S}\rangle$ is adjacent to $\mathrm{d}-1$ vertices in $\mathrm{S}$.

Therefore $X_{\text {icp }} \geq d-1$ $d X_{i c p} \geq\left(n-X_{i c p}\right)(d-1)=n d-n-d X_{i c p}+X_{i c p}$

i.e. $X_{i c p} \geq n\left(\frac{d-1}{2 d-1}\right)$

$$
n \leq X_{i c p}\left(\frac{2 d-1}{d-1}\right)
$$

Let Gi denote any i-regular on d-1 vertices where $d \geq 2$ and $2 \leq i \leq d-2$, both $\mathrm{d}$ and i have same property. 


$$
\begin{aligned}
& \text { When } \mathrm{i}=0,1,2 \quad \text { and } \mathrm{d}-1, \quad \text { then } \\
& G_{i} \cong K_{d-1}^{c},\left(\frac{d-1}{2}\right) K_{2}, C_{d-1} K_{d-1} \text { respectively. }
\end{aligned}
$$

\section{Theorem 1.4}

A d-regular graph of a graph $\mathrm{G}$ with $X_{i c p}=d-1$ iff $G \cong G_{i} V\left(\frac{d-1}{2}\right) k_{2}{ }_{\text {where }} 0 \leq i \leq d-2$

Proof: - Let G be A d-regular graph with $X_{i c p}=d-1$ and prove that $G \cong G_{i} V\left(\frac{d-1}{2}\right) k_{2}$ given that $\mathrm{G}$ is a dregular with $X_{i c p}=d-1$

$\Rightarrow \quad\langle\mathrm{S}\rangle$ has d-1 vertices and $\langle\mathrm{V}-\mathrm{S}\rangle$ has only independent edges.

By Theorem 1.3, $n \leq 2 d-1$ and sumce $\mathrm{G}$ is d-regular $n \geq d+1$

$\Rightarrow \quad d+1 \leq n \leq 2 d-1$

If $\langle\mathrm{V}$-S $\rangle$ has $\left(\frac{d-i}{2}\right)$ edges. Where $0 \leq i \leq d-2$ and both i \& d have same property.

$\Leftrightarrow \quad\langle\mathrm{V}-\mathrm{S}\rangle$ is 1-regular graph with d-I vertices.

$\Leftrightarrow \quad \mathrm{G}$ is d-regular (Every vertex in $\langle\mathrm{V}-\mathrm{S}$ ) is adjacent to all the $\mathrm{d}-1$ vertices in $\langle\mathrm{S}\rangle$ )

$\Leftrightarrow \quad$ Every vertex in $\mathrm{S}$ is adjacent to d-i vertices in $\mathrm{V}-\mathrm{S}$.

$\Leftrightarrow \quad$ Every vertex in $S$ is adjacent to exactly I vertices in S.

$\Leftrightarrow \quad\langle$ S $>$ is i-regular and S has d-1 vertices.

$\Leftrightarrow \quad<S>\cong G_{i}$

$$
<S>\cong G_{i} \vee\left(\frac{d-i}{2}\right) K_{2}
$$

\section{Illustration}

1) From these theorem and calculations authors conclude that

2-regular graph with $X_{i c p}=1$

3-regular graph with $X_{i c p}=2$

4-regular graph with $X_{i c p}=3$

5-regular graph with $X_{i c p}=4$
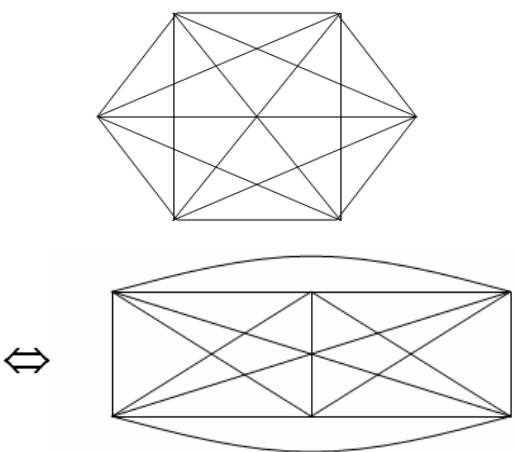

5-regular graph or K6

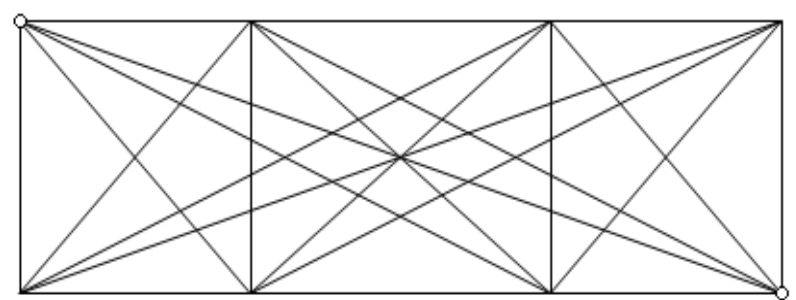

5-regular or $2 K_{2} \vee 2 K_{2}$

2) Theorem 1.2 and Theorem 1.4 gives the help of detection of planarity of Peterson graph.

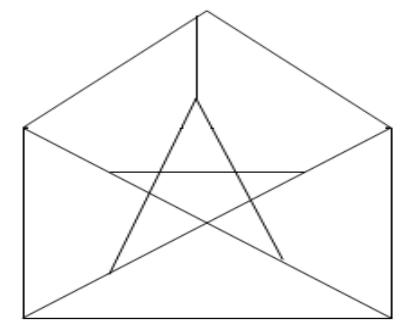

3-regular graph with $\mathbf{n = 1 0}$ is non-planner

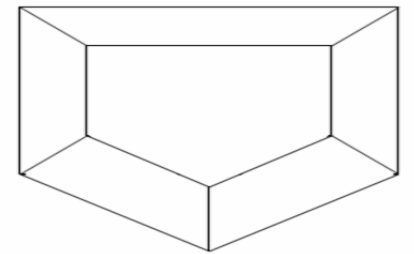

It is also a 3-regular graph but not Peterson graph

3) 6-regular Graph with $\chi_{i c p}=5$ 


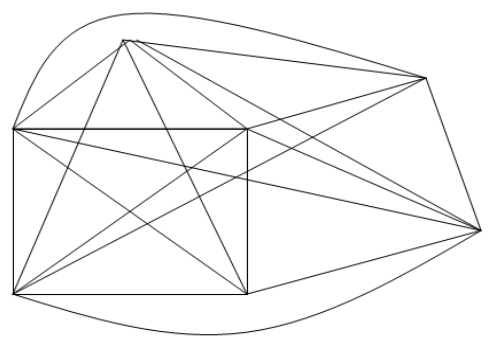

$\mathbf{K}_{5} \vee \mathbf{K}_{2}$

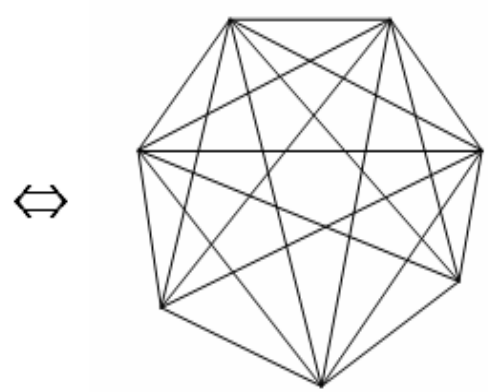

$\mathbf{K}_{7}$

\section{CONCLUSION}

In the conclusion part of this paper, we can say that we have successfully describe domination number of regular graphs as well as complimentary perfect domination number and induced complimentary perfect domination number, denoted by cpd and iced. In future we will try to do more in graph theory to prove few more facts about graphs.

\section{REFERENCES}

[1] Arumugam S, "Total Domination graph “ Arts Combing - 43 (2008) P.P / 89-92

[2] Paulraj Joseph, J and Arumugam S "Domination and connectivity in graphs. IJOMAS V-3 (1992) P.P 233-236

[3] Paulraj Joseph, J and Arumugam S "On graph with equal domination and connected domination number." Disc Math's 206 (1999)(45-49)

[4] Sampat Kumar, E Walikar, "The connected Domination number of a graph J. Math Physics science-13 (1979) No-6 P.P 607-613

[5] Teresa W, Hayes and Peter J. Slater Fundamentals of domination in Graph Journal of G.T. 071998

[6] Balakrishan R and Reganathan K, A textbook of Graph Theory, Springer Verlog, New York.

[7] Haynes, Teresa W, "Induced - Paired Domination n Graphs" Arts Combing 57 P.P (111-128)

[8] Salter, P.J "Domination and Reference set in a graph, J.Math Physicd Science” 22 (1998) P.P (455-465)

[9] W, maccuaig and B shepherd," Domination in graph with minimum degree two" Journal of graph Theory 13 (1989) (749-762).

[10] P,Manual,I Raja Singh J Quadras "Embedding of hypercube in to complete binary tree" ICICS,Iternational Conference, Saudi Arabia.(2000).

[11] Paulraj J Joseph and Mehadevan,"Induced and -paired domination chromatic number of a graph" Journal of Discrete Mathematics \& Cryptology.(Reprint) 